\title{
PIPE CRACKS DETECTION METHODS - A REVIEW
}

\author{
Ahmed Sachit Hashim ${ }^{1}$, Bogdan Grămescu², Constantin Niţu ${ }^{3}$ \\ 1,2,3 University POLITEHNICA of Bucharest \\ 313, Splaiul Independenței, 060042-Bucharest, Romania \\ E-mail: ahmedhashim774@yahoo.com
}

\begin{abstract}
A comparison between the most important methods for crack detection during external inspection of the pipes is presented. It is meant to choose the best suited one for robotic devices, aimed to perform the pipelines monitoring, because they are often under the action of the environmental stress factors, like high-temperature variation, especially met in the Middle East countries. As a native from the Republic of Iraq, the first author currently works at development of a robot for external inspection of pipes, within his $\mathrm{PhD}$ thesis, in order to provide full care to the pipelines, because they are non-replaceable. This way, their performance and reliability can improved and the production stopping is prevented.
\end{abstract}

Keywords: Crack Detection, Camera Detection, Acoustic Detection, Magnetic Field.

\section{Introduction}

Crack is one of the most common defects occurring in the metal parts, with particular consequences to fluid transport pipes. The cause of the cracks generation is the exposure of the metal to mechanical and thermal stress, resulting in a defect consisting in the separation of the internal crystals, due to fatigue. For pipes, this indicates there is a turbulent pressure inside, during operation, which leads to a slight change in their shape, resulting in gradual weakness and the appearance of cracks, which can grow larger over time.

The most important methods used to detect the pipes cracks, which can fit to a mobile robot for external pipe inspection are: computer vision systems (camera), magnetic field measurements and acoustic detection (microphone).

\section{Cracks Detection by Use of a Camera System}

Due to the high importance of this type of detection, it has attracted the attention of many scientists and researchers in the field, mostly for crack detection in the pipes that are subject to high temperature [1]. Image analysis techniques were also used to detect welding defects in pipelines [2], as recently proposed thermal image analysis system for nondestructive tests (NDT), during thermal stress tests [3].

The use of a highly sensitive system to detect small cracks (about 500 micrometers) [4] proved that detection of cracks based on thermal imaging in excitation methods depends on eddy currents [5].

Pulsed phase thermography and derivative techniques for cracks detection were also developed [6], while a laser technology for "active thermal imaging of the spot plane" was used [7]. This one is based on the local excitement of the point where the laser beam from the camera falls on the inspected pipe, similar to the analysis method used in the project Thermo Bot [8]. An example of cracks detection scheme by use of camera vision systems is presented in figure 1.

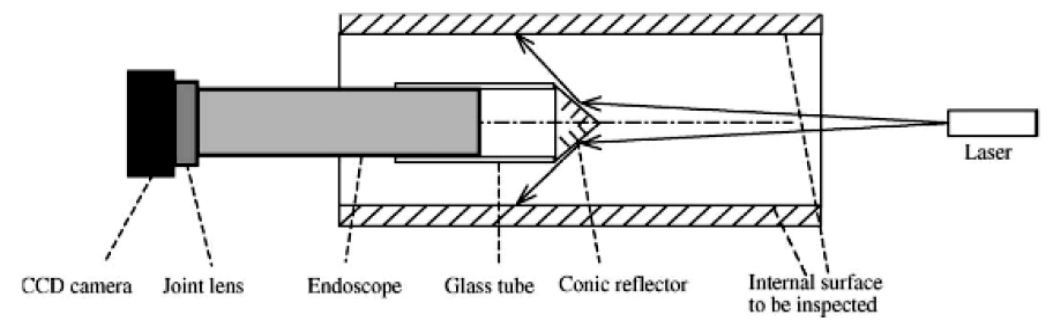

Figure 1: An industrial endoscope used for building a laser profilometry platform [18]. 
While the camera is working on the test of cracks in the pipes, a white spot surrounded by an area of low gray appears due to heat spread from the hotspot. The algorithm used to detect cracking of metal parts depends on the analysis of the area, where the heat is spread. The heat zone analysis is performed in three steps: hotspots detection, analysis of the radius of the pipe and tangential gradient analysis

As a first step, the hotspot should be detected on the pipe in the crack area. Because the laser spot is part of the top-temperature image, a simple algorithm can detect hotspots. A dynamic threshold is applied, depending on the lower and higher temperature values in the image, firstly to obtain a binary image, in which the laser position is the only white element. In the end, the center point is evaluated as the center of the mass of the resulting shape. Once the shape and location of the laser are available, it is possible to analyze the surrounding area to conduct detection of cracks in any smooth metal.

The first analysis involving the heat flow zone is performed on the radial gradient. While the normal regression factor takes into account the pixel difference only along the horizontal and vertical axes, the radial version takes into account multiple directions intersecting at a central point, corresponding to the hot point. Mid tones are usually evaluated by comparing points aligned along a given direction, intersecting with the center point. However, when evaluated in the discrete area of the image, one important side effect should be considered. The number of pixels in a given distance to the center is not constant, but depends on the distance itself. This is important in making comparisons. For example, in [18] it is suggested another approach instead of comparing pixel values, focusing on variable-sized image areas, which is based on distance to the entrance. Another slightly different approach to the problem is based on moving from the outside to the center. Consider the part of the image in the figure 2 (left). The center is green in the lower left corner. Per pixel, where the vector connecting it to the center appears, and its color is red if it crosses only one pixel, and blue if the pixels are intersecting two. It is important to separate these two cases in the form of pixels with a red vector that can be compared to another pixel (referred to by the vector), as in any other grading algorithm. Blue vector pixels need to be compared to more than one other pixel, the two types that intersect with the center vector, as shown in the figure 2 in the middle left, where each pixel is compared to more than two pixels.
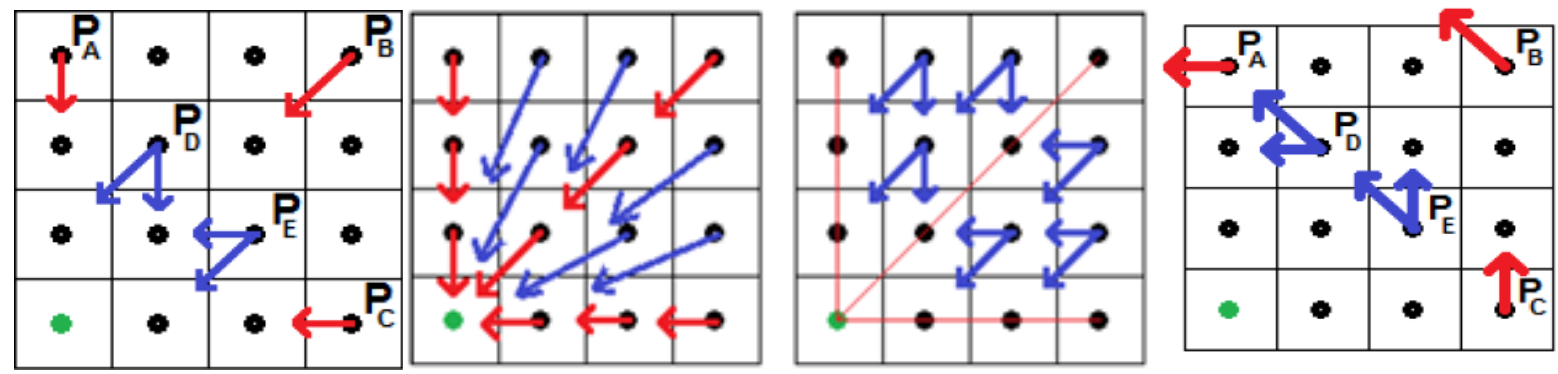

Figure 2: Four types about pixel comparison of the tangential gradient algorithm [18].

Consider the parts of the image with the increasing area described in [18] The use of the method described above can be identified in four different cases, which are summarized in figure 2.

The (PA, PB, and PC) pixels are connected to the center on a vertical, diagonal and horizontal direction respectively; therefore, the radial gradient is evaluated by comparing it to a single pixel, which is indicated by the vector. In the case of (PD), the line connected to the center of the image contains a

$$
\begin{aligned}
& P_{D}{ }^{\prime}=f\left(P\left(X_{D}-1, Y_{D}+1\right), P\left(X_{D}, Y_{D}+1\right)-P_{D}\right. \\
& P_{E}{ }^{\prime}=f\left(P\left(X_{E}-1, Y_{E}\right), P\left(X_{E}-1, Y_{E}+1\right)-P_{E}\right.
\end{aligned}
$$

where $P(X, Y)$ is the point of coordinates $(X, Y)$, and $f(\cdot)$ is a function that controls the different weight of adjacent pixels.

The gradient evaluated in the area around the laser one is not homogenous and is higher in stronger vertical component, therefore, the (PD) must be compared with the pixels placed along the left and right direction, (PE) is bright, so the values to be assumed are the following:

$$
\begin{aligned}
& P_{A}{ }^{\prime}=P\left(X_{A}, Y_{A}+1\right)-P_{A} \\
& P_{B}{ }^{\prime}=P\left(X_{B}-1, Y_{B}+1\right)-P_{B} \\
& P_{C}{ }^{\prime}=P\left(X_{C}-1, Y_{C}\right)-P_{C}(1)
\end{aligned}
$$


called radial gradient equalization (RGE) was proposed, which amplifies the gradient that depends on the distance to the center point of the laser region.

The equation is applied in a region surrounding the laser location until the maximum length specified by the parameter is reached.

Amplification is defined as a function of the distance to the center point and increases the linearity of the value until it reaches a certain value where it is accessed remotely, and then acquires saturation:

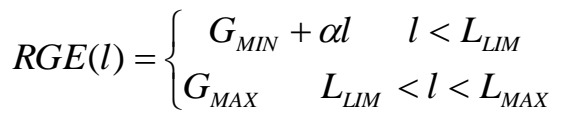

The results obtained using RGE can be seen in the figure 3, where the cracks are seen in the bottom edge after applying the equation in the direction right. The original picture, can be seen in the direction left.

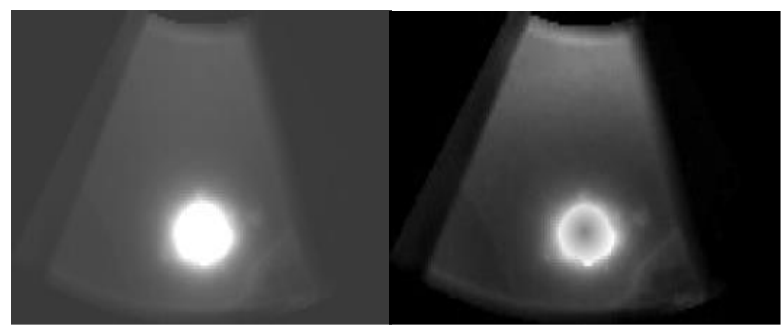

Figure 3: Comparison between the images before and after useof the radial gradient equalization equation (RGE) [18].

The proposed algorithm is designed to detect cracks by analyzing radial gradient images and has best results when adopting the equation (2). To detect cracks, the gradient image is divided into parts of small patches that are analyzed separately.

Each correction is numbered using an adaptive threshold, based on the average pixel value. Then, some image enhancement functions are applied, and finally, the cracks are detected by setting the features in the large size image, ignoring any image containing the spot of the laser.

The final detection algorithm for cracking is fairly simple, because it works on images that are strongly strengthened at the lower level.An example of detecting cracks can be seen in the figure 4 , where a binarized image is displayed in the left hand side with the final result in the right hand side.

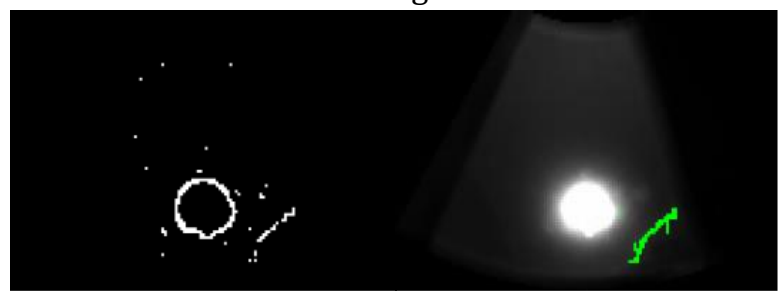

Figure 4: An example of image uniformity (left) and of the end result (right) of the used algorithm [18].
The tests of this algorithm included the detection of cracks in single images and full sequences. It is ideal for the case of smooth metal parts with circular shapes such as pipes. The tests showed a good performance of the algorithm, which was able to detect cracks in the area surrounding the laser fall site. This means that the cracks are detected when the mineral areas lie farther than the laser spot. The crack was very small, with a length of $8.36 \mathrm{~mm}$ and only 120 micrometers [18].

\section{Cracks Detection by Use of a Magnetic Field}

Magnetic field leakage (MFL) inspection is a nondestructive test (NDT) used to detect possible pipelines defects such as cracks, corrosion, drilling, scratches, pits, voids, etc. It is a wide-ranging method for detecting cracks in both axial and trans-verse directions.

MFL method is a more powerful one compared to the eddy currents method in terms of variation of magnetic properties.

The pulsed magnetic field leakage (PMFL) sensor system is designed to predict in the leakage field due to surface cracks and distant capillaries.

The accuracy and practical application of the MFL system have been experimentally evaluated for detecting surface and remote cracks of a sample made of low carbon steel, with a thickness of 10 $\mathrm{mm}[9]$. A large current around the pipe circumference was supplied, using the Helmholtz coil magnetization method to generate a strong magnetic field saturated around the wall of the pipe. This will result in high permeability in the magnetic infusion, which leads to allowing the sensors to detect the location and size of the cracks and how dangerous they are [10].

A small device has been studied and developed, to generate a magnetic field leakage with a sensor that acts as a receiver to detect cracks on high carbon steel pipes [11].

The test method for permeability measuring magnetic flux leakage PMFL was applied as a nondestructive (NDT)one, showing a good response to the crack shape. It is also sensitive to detecting deep defects, such as the internal or side cracks of the thick steel pipes.

More important, an electromagnet is used, flowing through the single coil, from a multi-track loop, where the loop produces a magnetic field required for detection [12].

Hall sensors were used to measure leaks in magnetic fields in the external pipes. Where that sensors receive the crack signal detection for the purpose of obtaining data and then send to the worker and to store and compress this data and reduce noise [13]. 


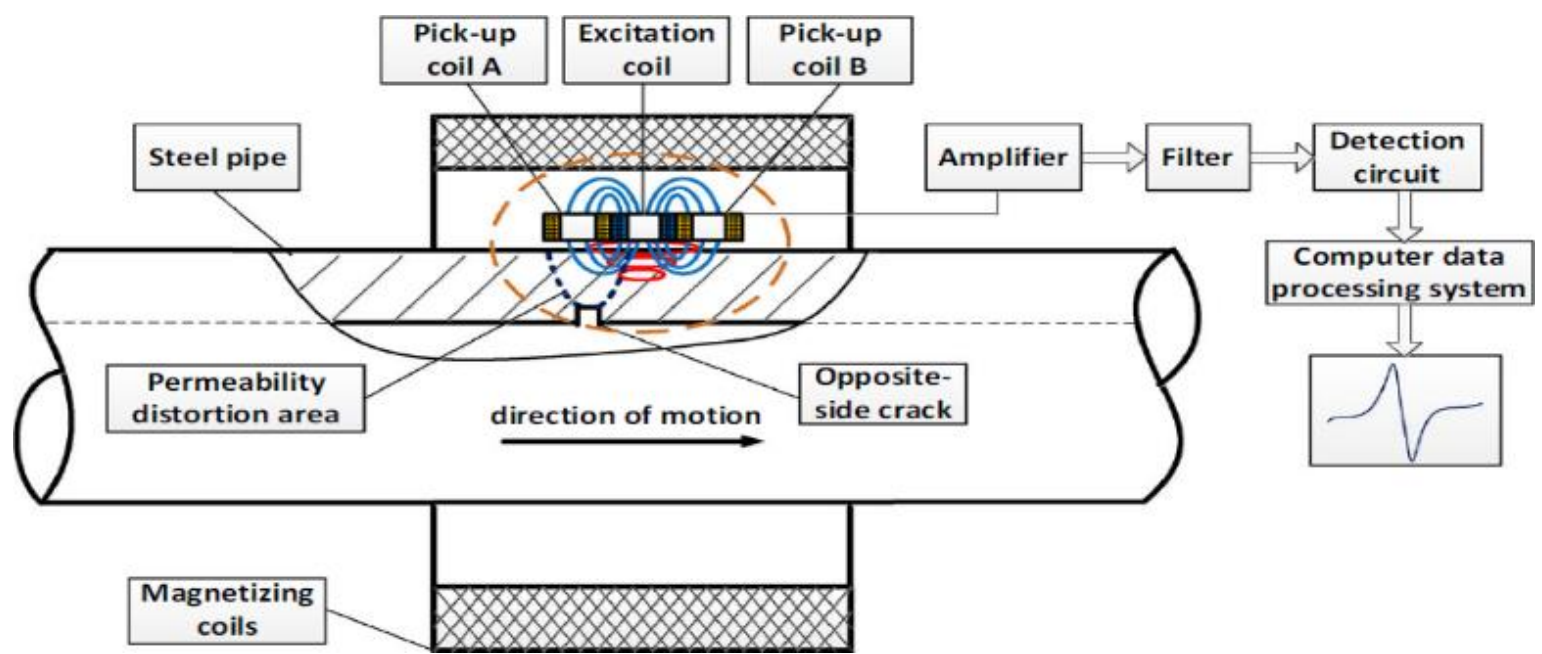

Figure 5: The schematic diagram of P-MFL testing method [12].

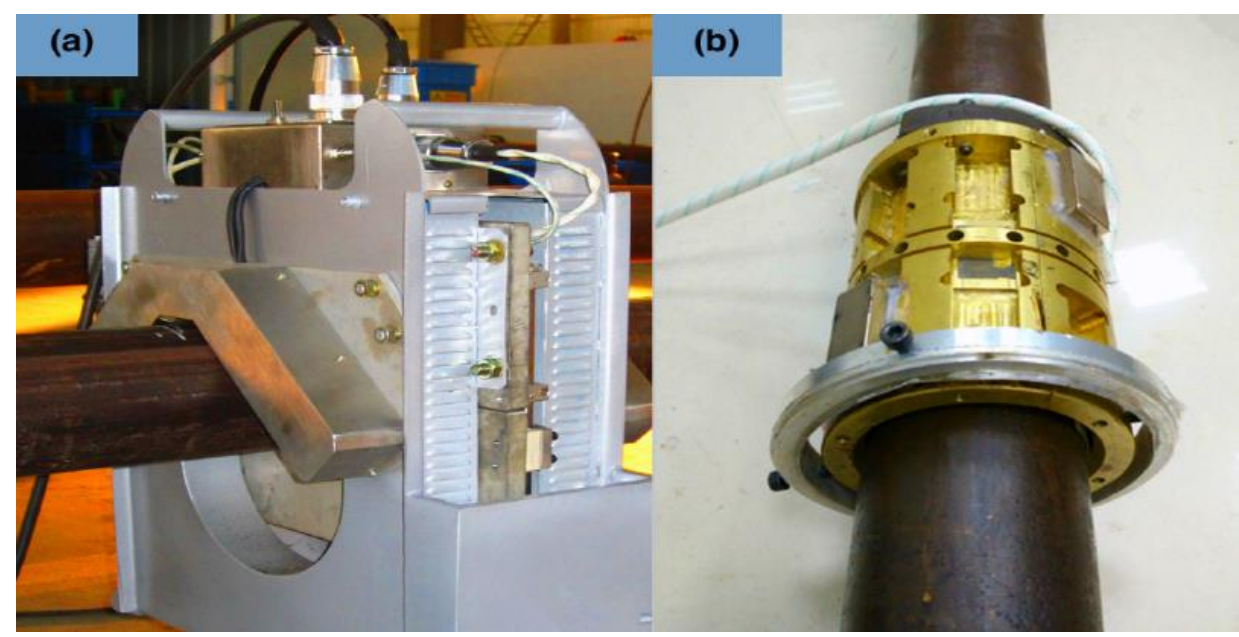

Figure 6: Practical application of P-MFL testing method [12].

The physical bases of the cracks detection in pipes, when using the magnetic field, depends on its distortion, when the inner surface cracked layer has a low permeability which increases towards the external surface of the pipe, as shown in the figure 7 , where the pipe usually reaches a magnetic saturation level.

The new P-MFL test method was analyzed and demonstrated by the simulations and experiments presented with good results. This method of inspection is similar to the eddy current test, but according to the principle of detection, it is quite different in detail because of the skin effect and the presence of insulating material around the pipes.

The detection limit in this P-MFL test exceeds 3 times the depth of the skin in the previous traditional way. However, for the P-MFL test method, the main difference is the fluctuations of permeability in the surface layer caused by deformation of the internal magnetic field lines. The proposed P-MFL test method differs from the previous MFL one from two very important points of view.
Firstly, although both benefit from the turbulent magnetic field caused by interruptions, the traditional methods measure the initial changes in the magnetic field, while the P-MFL test measures changes in the induced magnetic field, after interaction with the surface layer.

The MFL probe senses the physical quantities of the outer space, while the P-MFL test senses directly the property changes within the object. In addition, magnetization and captured signals are separated into different components and are perpendicular to space, but in the MFL test, the captured signals are part of the magnetic dimensional field.

Therefore, the P-MFL test method has a higher sensitivity and a large detection range, compared to the conventional MFL test method. To illustrate the advantage of the P-MFL method, a crack with depth 1 $\mathrm{mm}$, and $0.5 \mathrm{~mm}$ width was taken in a $14.5 \mathrm{~mm}$ thickness sample [12].

The figure 9 shows a typical component of magnetic flux density at a lift-off of $1 \mathrm{~mm}$.It can be seen that the amplitude value of $B_{\mathrm{MFL}}$ is only $2 \mathrm{mT}$, while the background magnetic density reaches 
nearly $225 \mathrm{mT}$, but the change in small magnetic density cannot be detected by ordinary magnetic sensors. Moreover, it is possible to immerse them in background noise, not to mention complex test conditions in practice. Figure 9 also shows the distribution of relative permeability above the crack.

This strong deformation of the permeability of the surface layer makes it easy to make a change in the distribution of the current and thus cause significant induced signals.

Secondly, because of the principle of interaction, magnetic pressure occurs while magnetic diffusion is disabled in a spatial area filled with a certain level of magnetic flow. Strong field input results in weak MFL outputs, resulting from the magnetic pressure effect (MCE) of the current MFL test sensor.

After that, an experiment was performed on the opposite crack system with a depth of $0.5 \mathrm{~mm}$ and 1 $\mathrm{mm}$ on the opposite side.

The test results showed that the P-MFL method was a good response to a $15 \mathrm{~mm}$ thickness pipe sample, while there were no clear signals by the conventional MFL method, under normal test conditions.

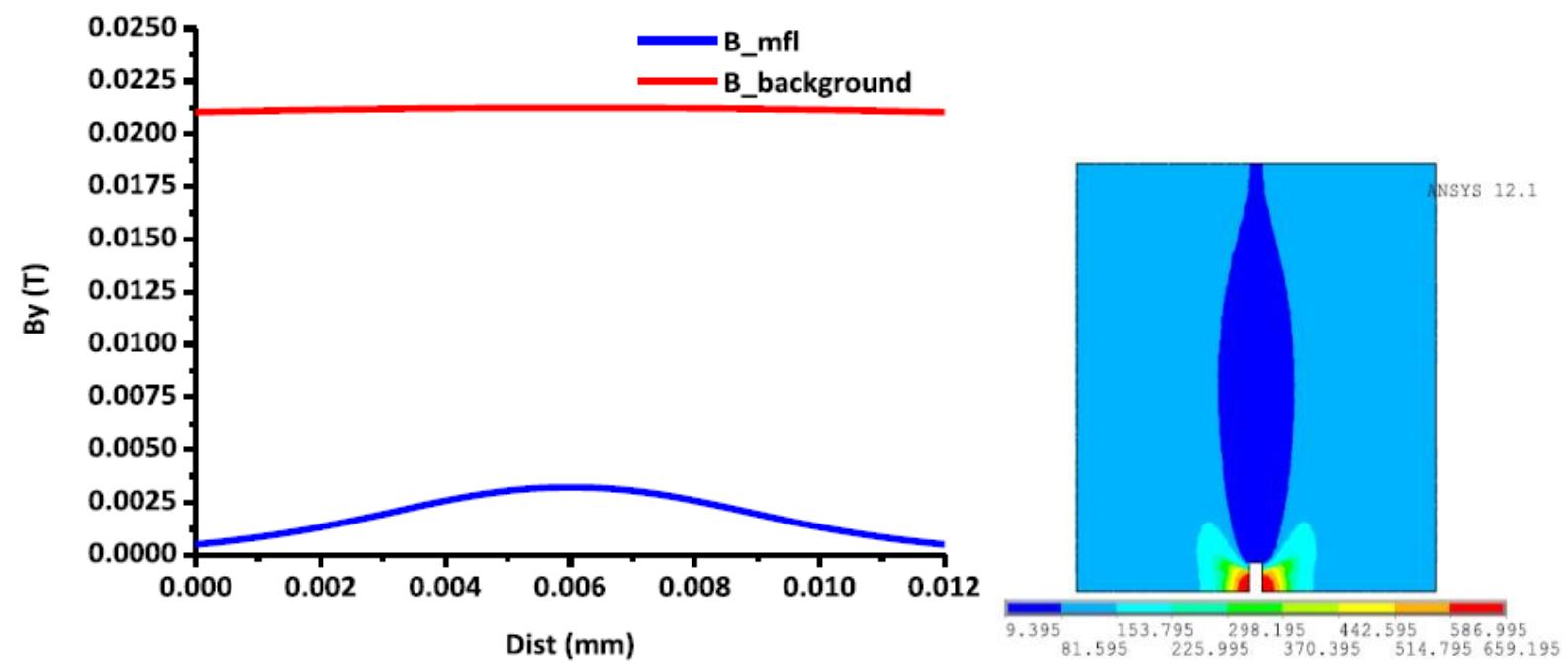

Figure 7: The magnetic leakage flux density $B_{y}$ (left) and relative permeability distribution, $\mu_{r}$ in P-MFL [12].

\section{Acoustic Detection of Cracks and Corrosion}

Probably, the newest method for detecting a crack with acoustic means was reported in [14]. The experiment was made on a pipe with a drilled hole, as artificial crack, to which an acoustic non-contact excitation was applied by help of laser-induced plasma (LIP) in air.

This is an effective way to produce intense sound, without damage of the tested structure. can realize non-contact acoustic impulse excitations.

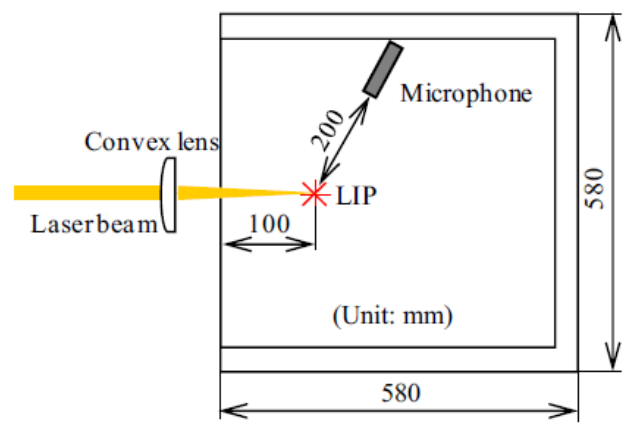

Figure 8: Acoustic excitation via LIP [14]

A microphone is used to measure the time response of the acoustic pressure, which differs if there is a crack/hole or not in the structure, due to the reflection of the acoustic wave. The position of the crack/hole is detected by applying the wavelet transform to the data regarding the measured time responses in the case of existing hole and not.

Stress wave propagation in solids (sounds and ultrasounds) is also used for crack detection. When cracks are produced and propagate, the high potential energy of deformation or fracture is released with acoustic emissions (AE).

$\mathrm{An}$ analysis of the detected $\mathrm{AE}$ waves related to cracks showed that they have frequencies in the range $50 \mathrm{kHz}-2 \mathrm{MHz}$, so piezoelectric sensors are required [15].

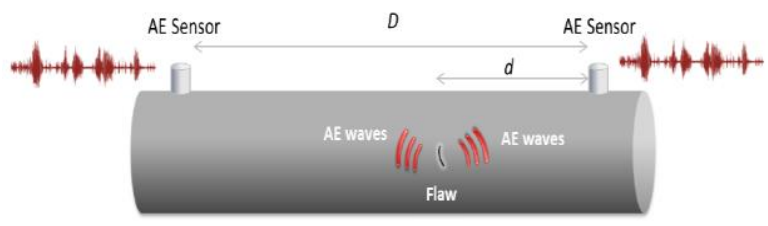

Figure 9: Detection of acoustic emission in a pipe [15].

As shown in figure 11, the flaw position can be determined if the time delay between hitting the first and second sensor, $\Delta \mathrm{T}$ is measured and the propagation speed is known for the pipe material (V). 
The distance between crack and fist hit sensor is:

$d=\frac{1}{2}(D-\Delta T . V)$

where $\mathrm{D}$ is the distance between sensors.

It is proved that the propagation of waves due to cracks in the gas pipelines also generate friction, which gives an important signal for the cracks detection. These results provide evidence that sound signals such as wave propagation and Hilbert Huang transform HHT are the best combination to detect cracks [16]. The analysis of acoustic technology is used to detect cracks, but also all obstructions by $10 \%$ of the area of the tube and the remote and continuous monitoring of oil and gas pipelines with large diameters [17].

\section{Conclusions}

By reviewing the most important literature references for three common and important methods for detecting cracks in pipes, relevant advancement was made for the future development of a robotic system for inspection and monitoring of the oil and gas pipes.

This is a challenge indeed, if considering the extreme possible environmental conditions as high temperatures in the Middle East countries, by taking into account that first author is from the Republic of Iraq. Therefore, the first type of detection, by use of a thermal camera is the best choice for flaw detection because it's easy to operate, has remote detection capabilities and good performance and reliability.

\section{References}

[1] Hwang, J., Kim, J., Lee, J.: Magnetic images of surface crack on heated specimen using an areatype magnetic camera with high spatial resolution, In: Instrumentation and Measurement Technology Conference,. I2MTC '09. IEEE, pp.1546-1551, IEEE 5-7 May 2009.

[2] Shafeek, H.I.,Gadelmawla, E.S., Abdel-Shafy, A.A., Elewa,I.M: Assessment of welding defects for gas pipeline radiographs using computer vision. NDT \& E International, 37 (4), 291-299 (2004), ISSN 0963-8695.

[3] Genest, M., Dudzinski, D. C., Bulmer, S., Kersey, R. K.: Crack detection using induction thermography for thermo mechanical fatigue tests. In: AIP Conf. Proc. 1335, pp. 1727-1734; doi: http://dx.doi.org/10.1063/1.3592137.

[4] Wagner, D., Ranc,N., Batthias, C., Paris, P.C.: Fatigue crack initiation detection by an infrared thermography method. Fatigue \& Fracture of Engineering Materials \& Structures, 33 (1), 12-21 (2010).
[5] Kostson,E., Weekes,V., Almond,D. P., Wilson,V., Tian,G. Y.: Crack detection using pulsing eddy current stimulated thermography. In: AIP Conf. Proc. 1335, pp. 415-422; doi:http://dx.doi.org/10.1063/1.3591882.

[6] Maldague,X.,Galmiche,F., Ziadi, A.: Advances in pulsed phase thermography. Infrared Physics \& Technology, 43 (3-5), 175-181 ( 2002), ISSN 1350-4495

[7] Maffren,T., Juncar,P., Lepoutre,F., Deban,G.: Crack detection in high-pressure turbine blades with flying spot active thermography in the SWIR range. In: AIP Conf. Proc. 1430, pp. 515-522; doi:http://dx.doi.org/10.1063/1.4716270.

[8] Ghidoni,S., Minella,M., Nanni,L., $\quad$ Ferrari,C., Moro,M., Pagello,E., Menegatti,E.: Automatic Crack Detectionin Thermal Imagesfor Metal Parts. Department of Information Engineering, University of Padova, Italy.

[9] Okolo.C.K, Meydana.T, :Pulsed magnetic flux leakage method for hairline crack detection and characterization. Wolfson Centre for Magnetics, School of Engineering, Cardiff University,UK, 2017.

[10] Wu.v, Fang. H, Li. L, Wang. J, Hang.X, Kang .Y, Sun .Y and Tang. C.:A Lift-Off-Tolerant Magnetic Flux Leakage Testing Method for Drill Pipes at Wellhead. Electronic Engineering, Newcastle University, UK, 2017.

[11] Ibrahim, M.Zakaria, Z., Aiman, A. M., Desmond, D.S., Nasir, M.A., Balkhis,I., Ruzairi,R. R.: Magnetic Flux Leakage Capsule Material Selection and Its Robustness Analysis in Oil and Gas Pipeline. Sensors \& Transducers, 156 (9), 67-74, (2013) Malaysia.

[12] Deng D., Sun, Y., Kang, Y., Song, K., Wang, R.: A Permeability-Measuring Magnetic Flux Leakage Method for Inner Surface Crack in Thick-Walled Steel Pipe. Springer Science, 5 September 2017.

[13] Shi,Y., Zhang,C., Li, R., Cai,M.,Jia, G.: Theory and Application of Magnetic Flux Leakage Pipeline Detection. Sensors, 15, 31036-31055 (2015)

[14] Kajiwara, I., Akita, R., Hosoya, N.: Damage detection in pipes based on acoustic excitations using laser-induced plasma. Mechanical Systems and Signal Processing (111) 570-579, (2018), Elsevier

[15] Integrity Diagnostics homepage http://www.idinspections.com, last accessed 2018/06/02

[16] Adnan. N. F., et al: Leak detection in the gas pipeline by acoustic and signal processing - A review. In: IOP Conference Series: Materials Science and Engineering, 2015.

[17] Tezerjani, A.D.: High-resolution visual pipe characterization system using an omnidirectional camera, $\mathrm{PhD}$ thesis, University of Regina, Saskatchewan; 2015.

[18] Jacquey,F.,Comby,F., Strauss, O.: Non-additive approach for omni directional image gradient estimation. France, 2007. 